\title{
System Identification and Data Fusion for On-line Adaptive Energy Forecasting in Virtual and Real Commercial Buildings
}

\author{
Xiwang $\mathrm{Li}^{\mathrm{a}, \mathrm{b}, *}$, Jin Wen ${ }^{\mathrm{a}}$ \\ ${ }^{a}$ Department of Civil, Architectural and Environmental Engineering, Drexel University, Philadelphia, PA 19104, USA \\ ${ }^{\mathrm{b}}$ Center for Green Building and Cities, Graduate School of Design, Harvard University, Cambridge, MA, 02138, USA
}

\begin{abstract}
Accurate, computationally efficient, and cost-effective energy forecasting models are essential for model based control. Existing studies in model based control have mostly been focusing on develop energy forecasting models using simplified physics based or data driven models. However, creating and identification the simplified physics model are often challenging, which requires expert knowledge for model simplification and significant engineering efforts for model training. In addition, the accuracy and robustness of data driven models are always bounded by the training data. To this end, developing high fidelity energy forecasting models with less engineering effort and good performance is still an urgent task. Although the previous studies from the authors have shown great promises and outperformed other data-driven and grey box models, they still have large errors at the special operation situations. Therefore, this paper investigates a novel methodology to develop energy estimation models for on-line building control and optimization using an integrated system identification and data fusion approach. The data fusion approach is able to adapt the forecasting model under the special operation situations. An eigensystem realization algorithm based model reformation method is developed to convert the system identification models into state space models. Kalman filter based data fusion techniques are then implemented on the state space models to improve the model accuracy and robustness. The developed methodology are evaluated using data from a virtual building (simulated) and a real small size commercial building. Three different data fusion intervals: 15, 30, and 60 minutes, have been tested. The

* Corresponding author. Tel.:+1 267563 0576; Email addresses: xiwang_li@gsd.harvard.edu (X. Li). jw325@drexel.edu (J. Wen)
\end{abstract}

(C) 2016. This manuscript version is made available under the Elsevier user license http://www.elsevier.com/open-access/userlicense/1.0/ 
overall building energy estimation accuracy from this proposed methodology can reach to above $95 \%$ in the virtual building and around $90 \%$ in the real building. The results also show that the shorter data fusion interval used, the higher accuracy can be achieved.

\section{Keywords}

Building energy forecasting, System identification, Data fusion, On-line estimation, Real field implementation

\section{Highlights}

1. Developed a novel real-time online adaptive energy forecasting methodology using system identification and data fusion

2. Proposed a modeling reforming method to reconstruct state space models from Markov parameters

3. Demonstrated the real field application of Kalman filter for parameter adaption and state estimation

4. Validated the proposed framework in a virtual and a real field commercial building

5. Achieved over $90 \%$ forecasting accuracy in both a virtual building and a real building 


\section{Nomenclature}

SID: system identification model

ERA: eigensystem realization algorithm

$S V D$ : singular value decomposition

$B A S$ : building automation system

$U$ : system inputs

$Y$ : system outputs

$P S D$ : power spectral density

CPSD: cross power spectral density

$C_{x y}$ : nonlinearity index

$S_{x x}$ : input variable auto power spectral density

$S_{y y}$ : output variable auto power spectral density

$S_{x y}$ : input and output variable cross power spectral density

$G(z)$ : transfer function in frequency domain

$G(t)$ : transfer function in time domain

$F_{\tau}:$ excitation signal

$a_{\tau}$ : excitation function magnitude scale parameter

$\omega_{\tau}$ : periodic frequency parameter

$T$ : sampling time

$\varphi_{\tau}$ : phase lag parameter

$A, B, C, D$ : state space parameter matrix

$w_{k}, v_{k}$ : forecast and measurement noise

$Q_{k}, R_{k}$ : forecast and measurement noise covariance

$K_{k}$ : Kalman gain

$P_{k}$ : estimate error covariance

$X_{k}:$ system state in state space models

$M P$ : Markov parameter

$H K$ : Hankel matrix

$E P_{k}$ : energy consumption from EnergPlus

$E(\cdot)$ : expected value

$R^{2}$ : coefficient of determination

$R M S E$ : root mean square error

1. NRMSE: normalized root mean square error 


\section{Introduction}

The electricity consumption of the US grew $1.7 \%$ annually from 1996 to 2006, and the total growth will reach $26 \%$ until 2030, among which buildings are responsible for over $70 \%$ [1]. Different technologies have been developed to reduce the building energy consumption and cost, such as better building design and retrofitting [2, 3], natural ventilation utilization $[4,5]$ and on-site distributed energy system integration $[6,7]$. Studies have shown that most of the commercial and residential buildings have operational problems that reduce the comfort and waste more energy. Around $4 \%$ to $20 \%$ of energy used in HVAC and lighting system is wasted due to equipment and operation problems [1]. Therefore the quality of building control and operation is significant economically and environmentally. Recent literature has proved that model based predictive control (MPC) approach has great potentials in improving building energy efficiency and reducing energy cost. The quality of MPC is significantly affected by the performance of building energy forecasting models [8]. A lot of building energy forecasting models have been developed in the past two decades from different perspectives. Li and Wen reviewed building energy forecasting models with emphasis on those used in building control and operation [9]. This review paper concluded that all the existing models: white box, grey box and black box models, have their own limitations in applying to real fields, either in engineering effort or uncontrollable robustness. One of the most comprehensive white box model for building energy estimation is EnergyPlus, which is a whole building energy simulation program that engineers, architects, and researchers use to model building performance [10]. Due to the detailed physics based equations regarding each component within buildings, it can achieve better accuracy and robustness. Two different MPC integration approaches have been developed to integrate EnergyPlus and optimization programs. The first approach is using "text-editing" software to edit "idf" file before the simulation engine's reading, and then the optimization program will evaluate the EnergyPlus output files and update the operation strategies. The second approach is using a middleware software to open a "sock" channel between the simulation tool and optimization engine. Therefore, the data exchanging can be realized at each time step 
during the simulation. The major difference between these two different coupling methods is that the first one can only exchange data after the simulation finished, while the second approach can exchange the information during the simulation. In [11], upon the MATLAB-EnergyPlus MPC environment, a particle swarm optimization (PSO) was adopted to search for near-optimal control for building energy system. This framework achieved 5\% cost saving by optimizing the hourly cooling setpoints in a large office building, and 54\% energy saving by optimizing hourly supply water temperature in a small office building. There also a lot of studies using GenOpt for building operation and control, such as [12, 13]. Besides the "text editing" approach, there are even more studies using the second approach to couple the building simulation models and optimization programing through BCVTB and MLE+. Yoon et al. studied the performance of MPC under the real-time electricity price [14]. This MPC achieved 9\% energy reduction and $14 \%$ electricity cost saving in a MLE+ based MATLAB and EnergyPlus environment. Using the same environment, Feng et al. investigated the performance of MPC for a radiant slab systems [15], which reduced cooling tower energy consumption by $55 \%$ and pumping power consumption by $25 \%$, comparing against an existing rule based control strategy. Even though these elaborated simulation tools are very accurate and robust, they require detailed information and parameters of buildings, energy system and outside weather conditions. Identifying these parameters, however, is very time consuming, costly, and needs expert knowledge. What is even more challenging, the simulation speed is relative low and not suitable to be used for on-line MPC.

Black box models, also known as data driven models, are typically more computational effective. For example, statistical methods, such as regression models (, autoregressive exogenous, Gaussian process regression, etc.) $[16,17]$, artificial neural network (ANN) [18], and support vector machine for regression (SVR) [19] are widely applied in existing studies to determine building control strategies to reduce energy consumption and energy cost. Besides using single data driven models, meta modeling approaches are also developed to forecast building energy consumption [20, 21]. However, such models often require long training period which sometimes can cause large forecasting errors when training data does not cover all the forecasting range. Grey box models, namely simplified physics based models, reduce the 
computational demand and still remain certain physics properties and require less effort in collecting training data comparing with black box models and in parameter determining comparing with white box models. Due to the model simplification, grey box models have shown higher computation efficiency than white box models. In addition, due to the physics based equations in the models, they also have shown better robustness than black box models. So they attract a lot of attention in MPC for building operation. Resistance and Capacitance (RC) network model is the most common grey box model for building load estimation. It has been used to estimate the building heating and cooling load [22, 23], as well as used to control building temperatures [24]. In the application of RC models, accurately determining the parameters is often the major challenge. Even though a lot of identification and optimization techniques provide applicable ways, determining the parameters of RC model is still computational demanding, as the model structures and parameters are unique from building to building and the equation in those models are complex and non-convex. As a result, even though numerus of energy forecasting models have been developed in the existing literature, there still is a lack of a general strategy to develop accurate, robust, and cost-effective energy forecasting models for building control and operation purposes. As a result, how to develop accurate, robust, and cost-effective energy forecasting models is the focus of this study.

Li and Wen $[25,26]$ therefore proposed to use system identification techniques, including active excitation schemes, to develop high fidelity building energy forecasting models without high engineering cost and with good scalability and extendibility. Instead of using a passive way for model development, system identification method collects the model training data in an active manner. The system identification (SID) method developed by Li and Wen has been applied in a real commercial building and has achieved with over $90 \%$ accuracy [27]. Unfortunately, the real field application results show that the model still has large uncertainties and errors at system changing periods, such as demand response operation situations. This observation is also one of the common shortcoming for most of the forecasting models. To overcome this shortcoming, this study proposes an on-line model adaptive approach to improve the models performance, especially under the special system operation conditions, using data 
fusion techniques. Data fusion has been applied in building energy forecasting studies due to its online updating capability. For example, the integration of grey box (RC) models with Kalman filter techniques has been studied in [28], as well as the combination of black box models and Kalman filters [29]. In all of the above studies, using Kalman filter technique has improved the overall model accuracy, although the inherited drawbacks of the grey box and black box models still cannot be resolved.

Therefore, the objective of this study is to integrate Kalman filter based data fusion techniques with our SID method to improve the forecasting accuracy and robustness. In the following sections, section 2 introduces the methods of SID model development and Kalman filter implementation briefly, followed by the description model performances evaluated using data from both virtual and real buildings in section 3 . Finally, section 4 discusses the results and achievements in the application in virtual and real buildings.

\section{Building Energy System Identification Model Development}

\subsection{SID model development}

The details about the SID model development can be found in [26]. Therefore, this section will only be a brief introduction. The selection of input and output variables are summarized in Table 1. Our SID model is developed to forecast cooling energy $\left(E_{\text {bldg }}\right)$ based on input variables such as outdoor air temperature $\left(T_{\text {out }}\right)$, Zone temperature setpoint $\left(T_{s t p, i}\right)$, etc. The model output, $E_{\text {bldg }}$, includes the energy consumption of chiller, chiller pump as well as the supply/return fans. The process of determining input feature utilizes correlation analysis and recursive selection. The general approach of the SID model is to use frequency response function to capture the dynamic relationship between system inputs and outputs. The results of the system identification results are saved as a set of Markov parameters. These Markov parameters will be used to create the state space model for Kalman filter implementation.

Table 1. SID model feature selection

\begin{tabular}{ccc}
\hline Variable & Variable Name & Type \\
\hline$E_{\text {bldg }}$ & Building cooling energy power $(\mathrm{kW})$ & Output \\
$T_{\text {out }}$ & Outdoor air temperature $(\mathrm{C})$ & Input
\end{tabular}




\begin{tabular}{ccc}
$T_{s t p, i}$ & Zone i temperature setpoint $(\mathrm{C})$ & Input \\
$R_{\text {in }, i}$ & Lighting/Equipment schedule in zone i (-) & Input \\
$Q_{d i r}$ & Direct solar radiation $\left(\mathrm{W} / \mathrm{m}^{2}\right)$ & Input \\
$Q_{d i f}$ & Diffuse solar radiation $\left(\mathrm{W} / \mathrm{m}^{2}\right)$ & Input \\
$V_{o a}$ & Ventilation rate $\left(\mathrm{m}^{3} / \mathrm{s}\right)$ & Input \\
\hline
\end{tabular}

After the model structure is developed, a system identification process is employed, which actively modifies several system operation parameters to generate training data that contain much richer information than typical building operational data. This process is referred to as system excitation hereafter. All the system inputs listed in Table 1 are categorized into excited inputs and unexcited inputs. The excited inputs are $T_{s t p, i}$ and $R_{i n, i}$. The other inputs are unexcited inputs, and they follow the regular building control schemes. The system excitation is a crucial step in this system identification approach for training data collection with considering the information entropy of the system operation. The system identification process applies excitation signals to generate the training data. Sum of sinusoids function is used to generate excited inputs (Eq. 1).

$$
F_{\tau+1}=F_{\tau}+\sqrt{2 a_{\tau}} \sin \left(\omega_{\tau} t T+\varphi_{\tau}\right) \quad \text { Eq. } 1
$$

Where $F_{\tau+1}$ is the excitation signal; $\sqrt{2 a_{\tau}}$ is a magnitude scale parameter from 0 to $1 ; \omega_{\tau}$ is periodic frequency parameter from 0 to $2 \pi$; $T$ is the sampling time, and $\varphi$ is the phase lag parameter from 0 to $2 \pi$. Again, more details about the excitation signal generation and evaluation can be found in [26].

\subsection{State space model development}

As introduced earlier, the SID model described previously is stored as a set of Markov parameters. In order to utilize Kalman filter for this SID model, however, a state space model is needed. The general linear discrete-time system state space model can be expressed as stochastic differential equations:

$$
\begin{gathered}
x_{k}=A_{k-1} x_{k-1}+B_{k-1} u_{k-1}+w_{k-1} \\
y_{k}=C_{k} x_{k}+v_{k}
\end{gathered}
$$


Where $x$ is the state, $u$ is the input, and $y$ is the measurement of the system. $w$ and $v$ are process and measurement noises, respectively. A, B, C, and D are state space parameter matrices, which need to be determined using the eigensystem realization algorithm (ERA) methods [30]. The overall procedure of state space model formation through ERA is illustrated in Figure 1.

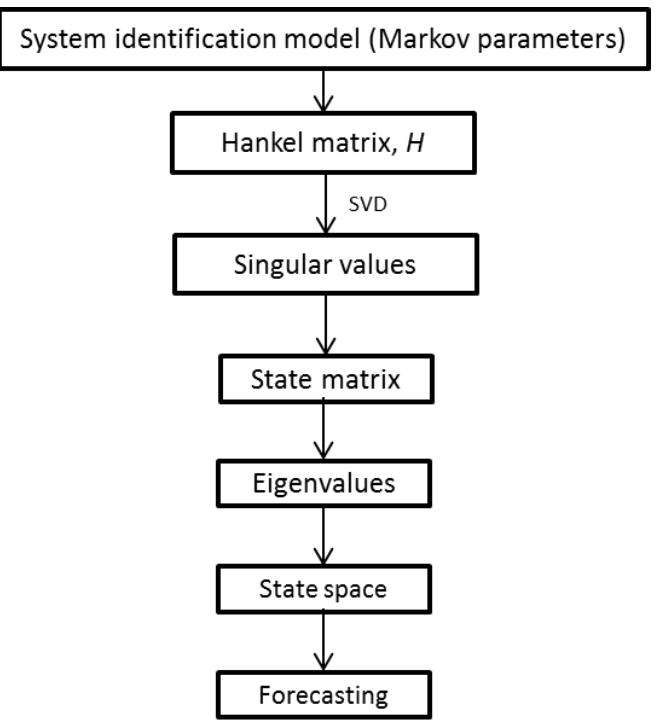

Figure 1. State space model through ERA

The details of Hankel matrix generation, state matrix formation and system order determining, etc. are introduced here. From Eq. 2 and Eq. 3, the Markov parameters can be expressed as:

$$
M P_{k}=C A^{k-1} B
$$

Eq. 4

All these Markov parameters $M P_{1}, M P_{2}, M P_{3}, \ldots$ are calculated in the SID model without explicit knowledge of the system matrices $A, B, C$, and $D$. The process of using ERA to convert SID to a state space model starts from a system realization matrix, Hankel matrix, which is composed of Markov parameters:

$$
H K(k-1)=\left[\begin{array}{cccc}
M P_{k} & M P_{k+1} & \cdots & M P_{k+\beta-1} \\
M P_{k+1} & M P_{k+2} & \cdots & M P_{k+\beta} \\
\vdots & \vdots & \ddots & \vdots \\
M P_{k+\alpha-1} & M P_{k+\alpha} & \cdots & M P_{k+\alpha+\beta-2}
\end{array}\right]_{\alpha m \times \beta r}
$$

Where $\alpha$ and $\beta$ are determined by the number of Markov parameters from the SID model developing process, both of which should be greater than system order $n$. 
Since building energy model is a dynamic model, whose system order could be changing according to the system operation situation (for example during starting up and shutting down period). Therefore, singular value decomposition (SVD) is then applied on $H K(k)$ to determine the system order and state space model parameters:

$$
H K(k)=R \Sigma S^{T}
$$

Where $\Sigma$ contains the singular values of $H$ on its diagonal, $R$ and $S$ are orthonormal matrices containing their corresponding singular vectors. The rank of $\Sigma$ is the order of this system model.

$$
\Sigma=\left[\begin{array}{cc}
\Sigma_{n} & 0 \\
0 & 0
\end{array}\right] \text { with } \Sigma_{n}=\operatorname{diag}\left[\sigma_{1}, \sigma_{2} \ldots \sigma_{n}\right]
$$

Therefore, the parameter matrices of the state space model can be obtained from the following equations:

$$
\begin{gathered}
\hat{A}_{k}=\Sigma_{n}^{-\frac{1}{2}} R_{n}^{T} H(k) S_{n} \Sigma_{n}^{-\frac{1}{2}} \\
\hat{B}_{k}=\Sigma_{n}^{\frac{1}{2}} S_{n}^{T} E_{r} \\
\hat{C}_{k}=E_{m}^{T} R_{n} \Sigma_{n}^{\frac{1}{2}}
\end{gathered}
$$

Where $E_{r}^{T}=\left[I_{r} O_{r} \ldots O_{r}\right]$ and $E_{m}^{T}=\left[I_{m} O_{m} \ldots O_{m}\right] . I$ is identical matrix, $O$ is identity matrix, $r$ is the number of inputs and $m$ is the number of outputs. $R_{n}$ and $S_{n}$ are the first $n$ columns of matrices $R$ and $S$, respectively. $\hat{A}_{k}, \hat{B}_{k}$ and $\hat{C}_{k}$ are the estimation of state space model parameters: $A_{k}, B_{k}$ and $C_{k}$.

In this study, a system order auto checking model has been implemented at each time step before state space model generation and Kalman filter implementation. In the state space model, state vector $X_{k}=$ $\left[E_{k} E_{k-1} \ldots E_{k-n}\right]$ ( $n$ is system order), measurement vector $Y_{k}=\left[E_{k}\right]$, and input vector contains all the input variables in the SID model, as described in Table 1 , where $E_{k}$ is building energy consumption forecasting from state space model. 


\subsection{Kalman filter implementation}

The concept of Kalman filter is to estimate the state of a dynamic system by using a feedback process: a state space filter estimates the state variables and receives feedback from noise measurements (Figure 2). The state space model will forecast forward (discrete time) from the current state and process covariance $Q_{k}$ to get a priori estimation of the state at next time step. The measurements with noises will be incorporated in the system model to update the posteriori forecasting. Such an adaptive process is able to capture the dynamics of building energy system, especially in the special operation situations with high uncertainties, such as demand response operation.

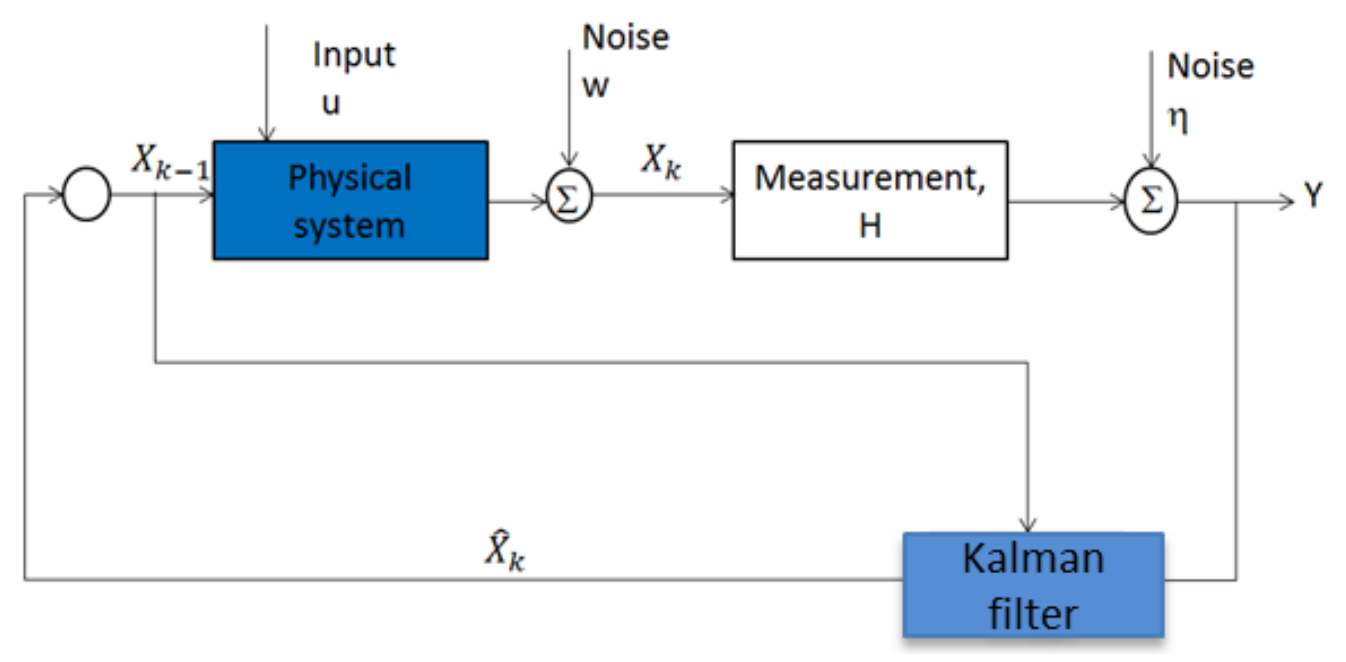

Figure 2. Kalman filter state estimation

Recall the state space equations in Eq. 2 and Eq. 3. Noises $w_{k}$ and $v_{k}$ have known covariance matrices $Q_{k}$ and $R_{k}$, respectively. $Q_{k}$ is determined by the discrepancy of the forecasted and real energy consumption. $R_{k}$ is determined by the measurement noise. This study assumes the normal distribution for process and measurement noises: $w_{k} \sim N\left(0, Q_{k}\right)$ and $v_{k} \sim N\left(0, R_{k}\right)$.

In this study, data from two buildings, namely a simulated building (virtual) and a real building, are used (details provided in Section 3.1). When the virtual building is used, simulation input and output variables from the EnergyPlus model are used as the ground truth (real energy consumption). A $\pm 10 \%$ measurement uncertainty (noise) is artificially added to the simulated ("measured") input/output 
variables. In the real building study, the real measurements are used and no artificial noise is added, as the real measurements are contains noises. Follow the Kalman filter implementation procedure discussed before, the process and measurement covariance are determined as:

$$
\begin{aligned}
& Q_{k}=I(n) * \sigma_{s s, k}{ }^{2} \\
& \text { Eq. } 10 \\
& R_{k}=\left(E P_{k} * \sigma_{m, k}\right)^{2}
\end{aligned}
$$

where $I(n)$ is a $n \times n$ identity matrix, $E P_{k}$ is the EnergyPlus cooling energy simulation results at step $k, \sigma_{s S}$ and $\sigma_{m}$ is the standard deviation of state space model and measurement errors, respectively. Then Kalman filter is applied to update the state space model and to improve the forecasting accuracy. The detailed steps about the Kalman filter algorithm implementation are provided in Table 2. In this study, three different updating intervals: 15 minutes, 30 minutes and 60 minutes, are tested.

Table 2 Kalman filter algorithm implementation

The state space model in Eq. 2 and Eq. 3 is a linear discrete time system. So the linear discrete time Kalman filter is used in this study. The algorithm is implemented as follows:

1. Starting from the state space model:

$$
\begin{aligned}
& x_{k}=A_{k-1} x_{k-1}+B_{k-1} u_{k-1}+w_{k-1} \\
& y_{k}=C_{k} x_{k}+v_{k}
\end{aligned}
$$

2. Define noises:

Determining forecasting error covariance, $Q_{k}$,

Determining measurement error covariance, $R_{k}$,

Process noises: $w_{k} \sim N\left(0, Q_{k}\right)$,

Measurement noises: $v_{k} \sim N\left(0, R_{k}\right)$

3. Initialization (as expected value $E(\cdot)$ ):

$$
\begin{aligned}
& \hat{x}_{0}^{+}=E\left(x_{0}\right) \\
& P_{0}^{+}=E\left[\left(x_{0}-\hat{x}_{0}^{+}\right)\left(x_{0}-\hat{x}_{0}^{+}\right)^{T}\right]
\end{aligned}
$$

4. Propagating:

Prior state estimation: $\hat{x}_{k}^{-}=A_{k-1} \hat{x}_{k-1}^{+}+B_{k-1} u_{k-1}$

Posteriori state estimation: $\hat{x}_{k}^{+}=\hat{x}_{k}^{-}+K_{k}\left(y_{k}-H_{k} \hat{x}_{k}^{-}\right)$ 
Where,

Kalman gain factor: $K_{k}=P_{k}^{-} H_{k}^{T}\left(H_{k} P_{k}^{-}+R_{k}\right)^{-1}$

Prior covariance: $P_{k}^{-}=A_{k-1} P_{k-1}^{+} A_{k-1}^{T}+Q_{k-1}$

Posteriori covariance: $P_{k}^{+}=\left(I-K_{k} C_{k}\right) P_{k}^{-}$

\section{On-line Building Cooling Energy Forecasting Development and Discussion}

\subsection{Building description}

This study implements the proposed on-line forecasting methodology in both a virtual and a real commercial building. The first one is a EnergyPlus simulation model, provided by U.S. Department of Energy (DOE) [31], in lieu of a real building. This building is a three-story office building, and each floor has five conditioned zones (Figure 3). This building uses multi-zone variable-air-volume (VAV) system with electric reheat. The VAV system is served by a chiller with $414 \mathrm{~kW}$ capacity and a nominal COP of 2.8. The location of this building is Philadelphia.
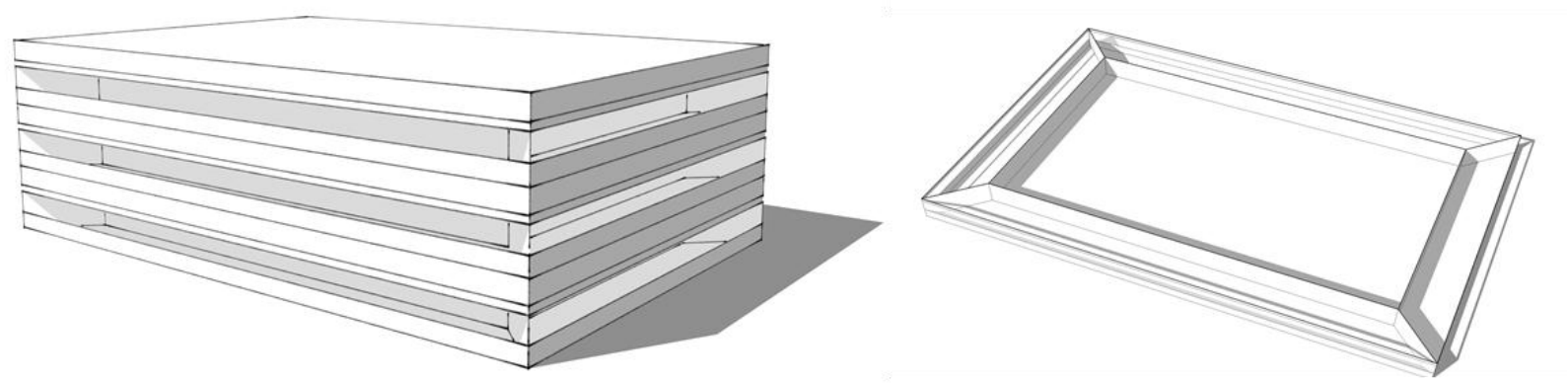

Figure 3. Building view and floorplan of DOE medium office building The other one is a real small size commercial building at the Energy Resource Station (ERS) of Iowa Energy Center. The ERS building is a small size commercial building with experiment area and common office area. The percentage of exterior window area to exterior wall area is $54 \%$ for each exterior zone. The zone thermometers are located on the center of the internal wall (shown as the blue box on the floor plan in Figure 4). The experiment area has two full-scale commercial HVAC systems with similar internal loads. The schematic diagram of the floor plan for the ERS is shown in Figure 4. The facility is equipped with three VAV air handling units (AHU). AHU-1 serves the common areas of the building. The remaining 
two AHUs serve the A- and B-Test Systems. AHU- A and B are identical, with each AHU serving four zones (three exterior and one interior). Both of these two AHUs are equipped with dual (supply and return) variable speed fans and are operated similarly to those in typical commercial buildings. Each testing system is equipped with a ten-ton air cooled chiller. Each test room has lighting systems (two operation stages) and baseboard heaters (two operation stages, each is 900W). Table 3 summarizes the key parameters for the building construction and HVAC system. More details about this experiment facility and real building implementation can be found in [27, 32].
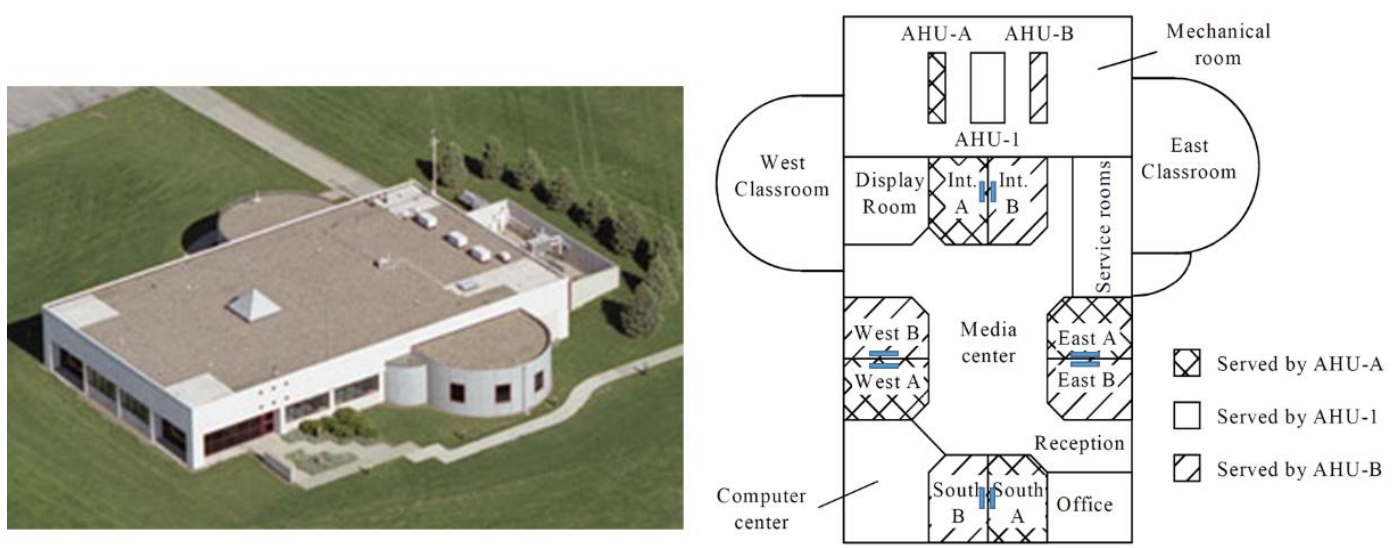

Figure 4. Building view and floorplan of ERS [32]

Table 3 Building description and HVAC features

\begin{tabular}{ccc}
\hline Feature & Medium Reference & Real small \\
\hline Floors & 3 & 1 \\
Total floor area $\left(\mathrm{m}^{2}\right)$ & 4982 & 855.5 \\
Window to wall $\mathrm{ratio}^{2}$ & 0.33 & 0.54 \\
Glazing U-factor $\left(\mathrm{W} /\left(\mathrm{m}^{2} \cdot \mathrm{K}\right)\right)$ & 3.35 & 2.73 \\
Glazing SHGC & 0.36 & 0.85 \\
Occupant Density $\left(\mathrm{m}^{2} /\right.$ person $)$ & 18.6 & 12.4 \\
Lighting Power Density $\left(\mathrm{W} / \mathrm{m}^{2}\right)$ & 16.89 & 6.88 \\
Equipment Power Density $\left(\mathrm{W} / \mathrm{m}^{2}\right)$ & 10.8 & 8.1 \\
HVAC & Packaged MZ VAV & AHU + VAV \\
\hline
\end{tabular}

\subsection{Training and testing condition}

In the virtual building, EnergyPlus model was simulated for two different period: 1) from August $1^{\text {st }}$ to August $7^{\text {th }}, 2$ ) from August $22^{\text {nd }}$ to August $28^{\text {th }}$. Simulated building operation data during time period 1 is used as training data for the SID model. The operation data during the second time period was for 
model validation. The reason why there is one week gap between these two periods is to bring in operation condition differences and to show the capability of the proposed on-line adaptive scheme is able to guarantee the forecasting performance under forecasting conditions different to training conditions. The system excitation signals discussed in section 2.1 was generated in MATLAB. In order to apply the system excitation signals into EnergyPlus model, a building cluster operation emulator was used [33]. As EnergyPlus is a deterministic simulation tool, no uncertainty is considered in the simulated building data, two sets of simulated data were generated in the virtual building measurements to better represent the real field situation. One set of data without measurement noise was labeled as noise-free data in the following sections. The other set of data with measurement noise, by adding Gaussian distributed random white noises to each measurement, was labeled as noisy data.

The real field implementation was conducted from $08 / 25 / 2015$ to $09 / 06 / 2015$. The training period was from 12:00 am 08/31/2015 to 9:00 pm 09/01/2015, and 9:00 pm 09/02/2015 - 12:00 am 09/05/2015. The forecasting and data fusion period was from 12:00 am 09/06/2015 - 12:00 am 09/06/2015. Both sunny and cloudy days were included in the training period. The outside temperature range at the training period was $16.5{ }^{\circ} \mathrm{C}$ to $32.7{ }^{\circ} \mathrm{C}$, while the temperature range at the forecasting period was $20.5{ }^{\circ} \mathrm{C}$ to $31.2^{\circ} \mathrm{C}$. The forecasting day was a sunny day. Different from the case in the virtual building, no artificial noises were added to the real field measurements, since the noises already existed in the measurements. Excitation signals were applied in the training period of both virtual building and real building studies. Figure 5 illustrates one sample excitation signals for model training (Figure 5a) and building operation schemes for forecasting validation (Figure $5 b)$. 

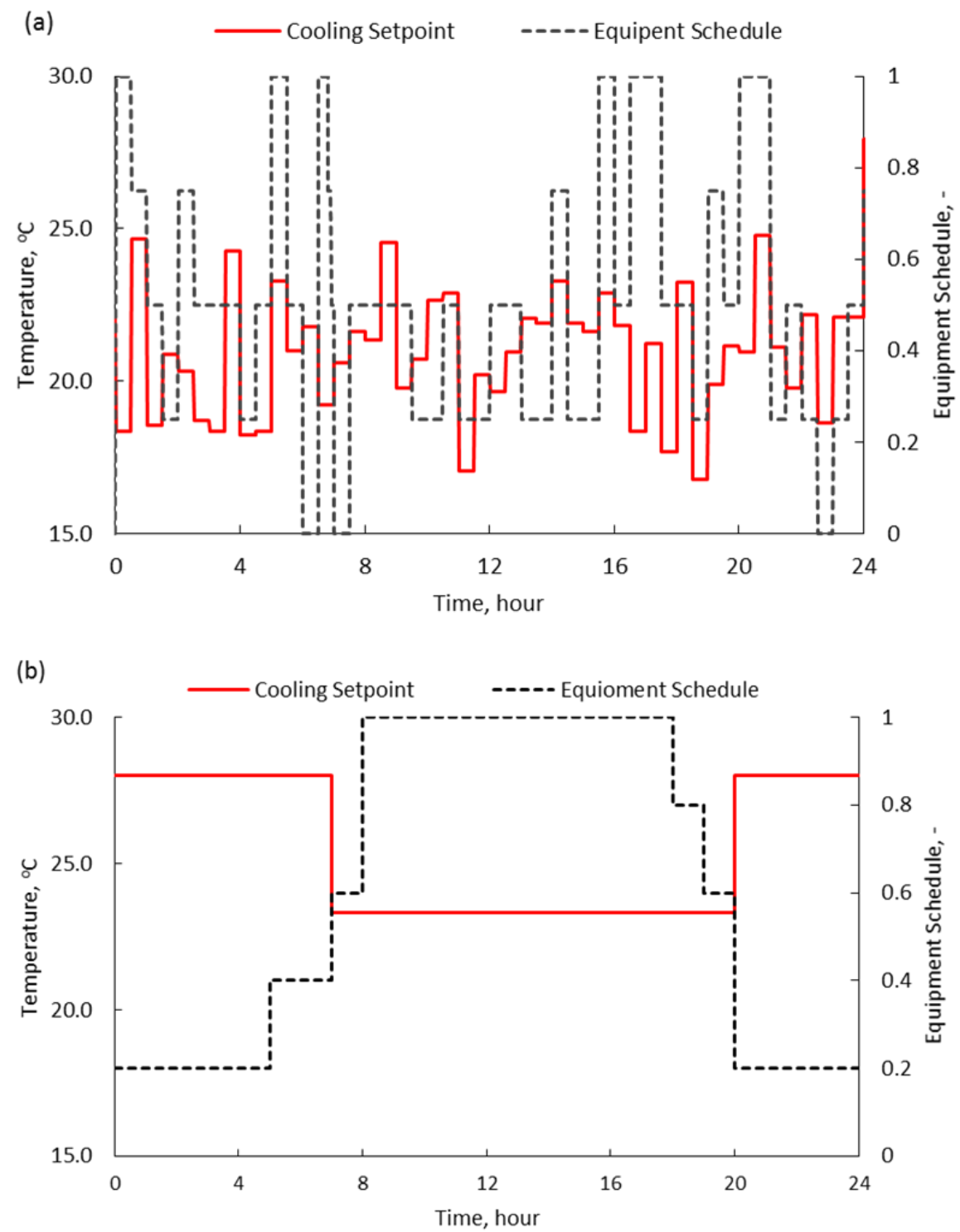

Figure 5. Operation signals for excitation and validation

\subsection{Building energy on-line estimation}

Figure 6 illustrates the overall procedure of the development and validation of the on-line adaptive energy forecasting model. In summary, there are four stages in the procedure: 1) system excitation stage, where model training and validation data is generated under the designed system excitation operation schemes; 2) system identification stage, where SID model is developed based on the system operation data; 3) state space model reforming stage, where SID model is converted to the state space model; 4) Kalman filter on-line estimation stage, where energy consumption is forecasted based on the measurements (in the virtual building case, the simulated measurements) at each time step. Both virtual building and real building cases follow the same procedure. In the real field on-line forecasting stage, all 
the real measurements are collected from the building automation system. Then the Kalman filter model updates and adapt the energy forecasting model based on the measurements from the real building to improve the model accuracy and robustness at each time step. The validation data and measurements are building operation data under the typical building operation schemes. As introduced in section 3.2, noises are artificially added to all of the measurements collected in the virtual building.

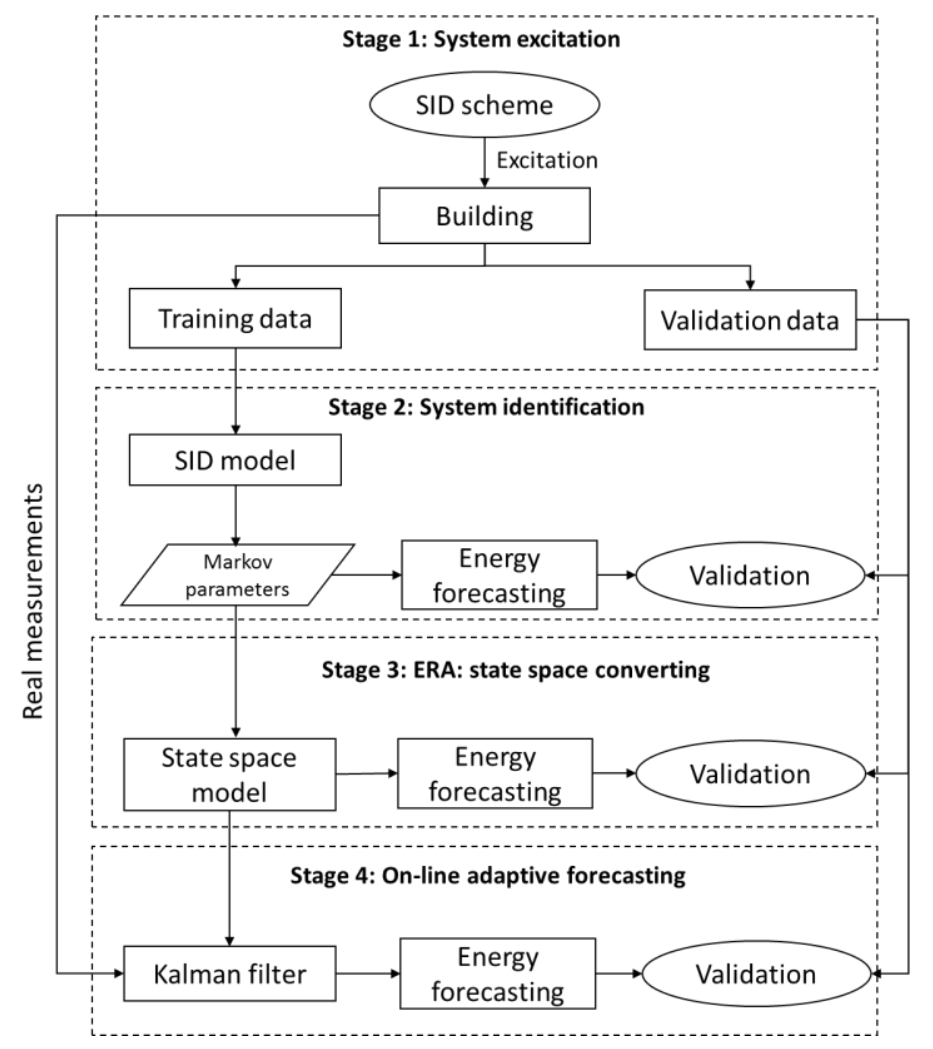

Figure 6. On-line model development procedure

\section{Results and Discussion}

\subsection{Model performance indices}

In order to evaluate the model performance, four indexes are employed, namely, Coefficient of Determination $\left(\mathrm{R}^{2}\right.$ ), Root Mean Square Error (RMSE), Normalized Root Mean Square Error (NRMSE), and Fractional Bias (FB):

$$
R^{2}=\frac{\sum_{i=1}^{n}\left(x_{i}-\bar{x}\right)\left(\hat{x}_{i}-\overline{\hat{x}}\right)}{\sum_{i=1}^{n}\left(x_{i}-\bar{x}\right)^{2} \sum_{i=1}^{n}\left(\hat{x}_{i}-\overline{\hat{x}}\right)}
$$




$$
\begin{gathered}
R M S E=\sqrt{\frac{\sum_{i=1}^{n}\left(x_{i}-\hat{x}_{i}\right)^{2}}{n}} \\
N R M S E=\sqrt{\frac{\sum_{i=1}^{n}\left(x_{i}-\hat{x}_{i}\right)^{2}}{n}} /\left(x_{\max }-x_{\min }\right) \\
F B=2 \sum_{i=1}^{n} \frac{\hat{x}_{i}-x_{i}}{\hat{x}_{i}+x_{i}} / n
\end{gathered}
$$

Where $x_{i}$ and $\hat{x}_{i}$ is the real and forecasting value; $\bar{x}$ and $\bar{x}$ are the average of measured and forecasted values, respectively. $x_{\max }$ and $x_{\min }$ are maximum and minimum of the measured energy consumption. The FB will have a value of 0 when $x_{i}$ and $\hat{x}_{i}$ match perfectly and will tend towards -2 or 2 as these quantities differ by greater magnitudes. If $\mathrm{FB}$ is greater than 0 , it indicates that the model tends to overestimated the system, and vice versa.

\subsection{Virtual building study results}

As introduced in section 2.1 and 3.2, the SID model is trained on the operation data from August $1^{\text {st }}$ to August $7^{\text {th }}$, and is saved as set of Markov parameters for each input. Then the Markov parameters for each inputs are used in ERA for state space model development and Kalman filter for data fusion. The energy forecasting results for each model are discussed in this section. In this section, three different forecasting and Kalman filter updating intervals: 15, 30 and 60 minutes are studied.

1. Case I: 15 minutes interval

In this case, the noisy training and validation data are collected every 15 minutes. Figure 7 plots the energy forecasting results under 15-mintues time step forecasting case. In this figure, the SID state space model (blue line) has large fluctuations in the forecasting results and tends to overestimate the energy consumption, especially at the weekends (the last two days). Kalman filter results (black line) are very close to the "measured" values with slight underestimation. The detailed performances of these three models are summarized in Table 4. Generally speaking, the state space model has the lowest accuracy with $\mathrm{R}^{2}$ as 0.76 . There are two potential causes for this decreased accuracy: 1) the truncating error in the state space model reformatting process, 2) the effect of the noise added into the measurements. However, 
the application of Kalman filter improves the accuracy to be over $90 \%\left(\mathrm{R}^{2}\right)$ and reduces error to be below $10 \%$ (RMSE $=6.6 \mathrm{~kW}$ and NRMSE=9\%) under noisy conditions. The FB index is above zero for the state space model, which means that the state space model tends to overestimate the energy consumption. FB results from the Kalman filter models are closer to zero than the other two models.

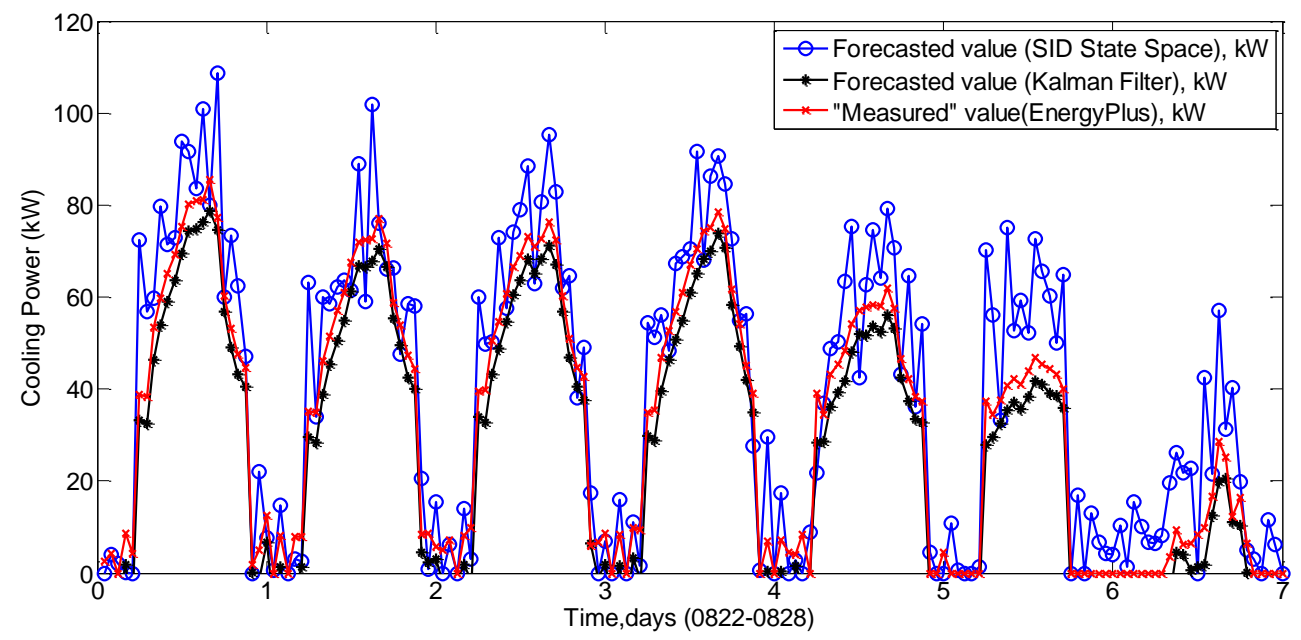

Figure 7. Virtual building energy forecasting model calibration results: 15 minutes

2. Case II: 30 and 60 minutes intervals

In these following two cases, all the conditions, such as forecasting period, forecasting models, weather conditions, indoor operation conditions, etc. are all remained the same as Case I, except the time interval. The following figure (Figure 8) plots the energy forecasting results for virtual building energy forecasting. The upper plot Figure 8a is the forecasting results with 30-minute forecasting interval, and the lower one (Figure 8b) is forecasting results with 60-minute forecasting interval. In these two plots, both state space model and Kalman filter model are under noisy condition. These two plots clearly show that the state space model (blue line) is still able to capture the trend of the energy consumption and can achieve good accuracy in the forecasting. The fluctuation of the state space results is larger than that of the Kalman filter model (black) due to the uncertainties in the measurements. Especially at the "Starting up" periods (when the building is changing from unoccupied to occupied), the state space model overestimates the energy consumption largely, while using the Kalman filter improves the forecasting 
accuracy to be over $0.9\left(\mathrm{R}^{2}\right)$ and the error to be below $10 \%(\mathrm{RMSE}=6.9 \mathrm{~kW}$ and NRMSE $=9 \%)$ for the case with 30-mintue intervals. For the case with 60-mintue intervals, the forecasting accuracy is still acceptable with $\mathrm{R}^{2}=0.86$, and $\mathrm{NRSE}=11.2 \%$. The spikes at the starting up period in the state space models are believed to be caused by the process noises, as artificial noises are added into the virtual building forecasting scenarios. The FB results in these two cases are similar to those in the case with time step as 15 minutes. The SID models underestimate, the state space models overestimate the energy consumption, and the Kalman filters improve the accuracy and robustness significantly.
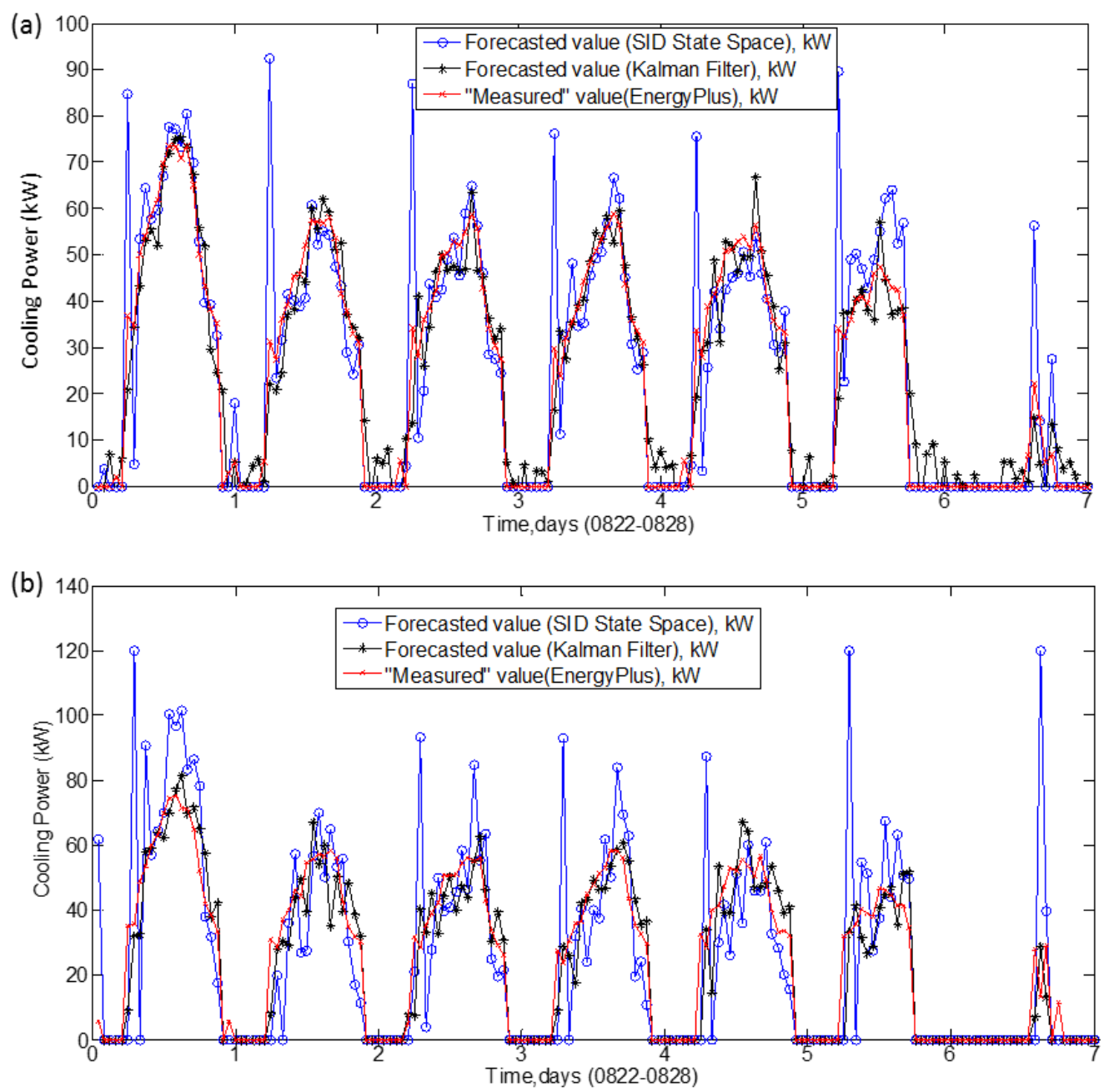

Figure 8. Virtual building energy forecasting model calibration results

The detailed forecasting accuracy statistics for all the models with all these 3 intervals are summarized in Table 4, which clearly shows that the models with shorter time interval are able to achieve 
higher accuracy, and Kalman filter models have the highest accuracy comparing with SID models and State space models. In all, the test results show that the SID and Kalman filter based energy forecasting model are able to improve the model accuracy and robustness under noisy conditions.

Table 4. Virtual building energy forecasting accuracy comparison

\begin{tabular}{cccccc}
\hline $\begin{array}{c}\text { Interval, } \\
\text { min }\end{array}$ & Model & $\mathbf{R}^{\mathbf{2}}$ & RMSE, $\mathbf{k W}$ & NRMSE & FB \\
\hline \multirow{2}{*}{15} & SID (noise free) & $\mathbf{0 . 9 2 *}$ & $\mathbf{5 . 9}$ & $\mathbf{8 \%}$ & -0.17 \\
& State Space (noisy) & 0.76 & 16.8 & $18 \%$ & 0.19 \\
& Kalman Filter & $\mathbf{0 . 9 2}$ & 6.6 & $9 \%$ & $\mathbf{- 0 . 1 4}$ \\
\hline \multirow{2}{*}{30} & SID (noise free) & 0.82 & 10.9 & $14.0 \%$ & -0.21 \\
& State Space (noisy) & 0.72 & 12.5 & $15.3 \%$ & 0.14 \\
& Kalman Filter & $\mathbf{0 . 9 1}$ & $\mathbf{6 . 9}$ & $\mathbf{9 \%}$ & $\mathbf{0 . 0 6}$ \\
\hline \multirow{2}{*}{60} & SID (noise free) & 0.76 & 12.9 & $15.0 \%$ & -0.25 \\
& State Space (noisy) & 0.69 & 13.4 & $18.0 \%$ & 0.24 \\
& Kalman Filter & $\mathbf{0 . 8 6}$ & $\mathbf{8 . 7}$ & $\mathbf{1 1 . 2 \%}$ & $\mathbf{0 . 1 0}$ \\
\hline
\end{tabular}

*Highest accuracy $\left(\mathrm{R}^{2}\right)$, lowest error (RMSE and NRMSE), and FB indexes closest to zero are bold

\subsection{Real field study results}

The system identification, state space model reformation, and data fusion approaches introduced in section 2, are also used to develop the energy forecasting models for the real commercial building introduced in section 3.1. Details about how this building is excited and how building data are collected can be found in section 3.2 and in [27]. Again, three data fusion intervals, i.e. 15 minutes 30 minutes, and 60 minutes, are used here.

1. Case I: 15 minutes data fusion intervals

Figure 9 plots the cooling energy forecasting results from the forecasting models with 15-mintue intervals. As shown in this figure and the statistics summary (Table 5), the state space model (blue) underestimates the cooling power and the Kalman filter improves the forecasting accuracy from $0.79\left(\mathrm{R}^{2}\right)$ to $0.92\left(\mathrm{R}^{2}\right)$. Comparing to the original SID model, the forecasting accuracy $\left(\mathrm{R}^{2}\right)$ of the SID state space model is decreased from 0.85 to 0.79 . Although there are no artificial noises added, real measurement 
noises exist, which could contribute the accuracy reduction. Another reason for the decreased accuracy is the truncating error in the state space model generation process.

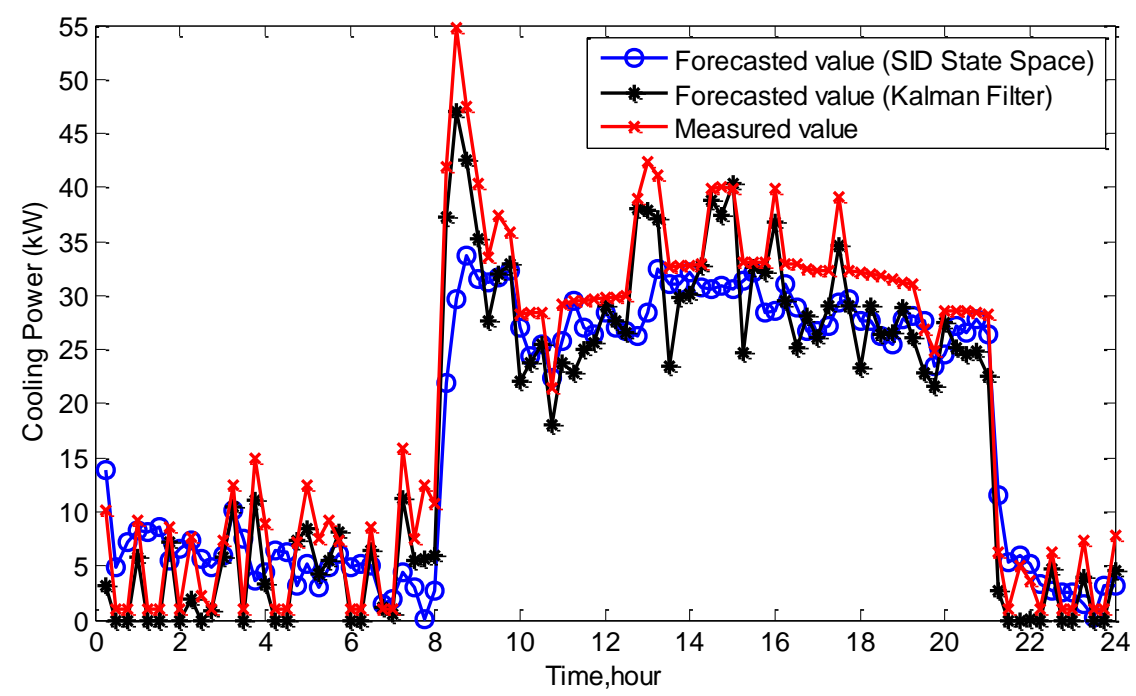

Figure 9. Real building energy forecasting model calibration results: 15 minutes

2. Case II: 30 and 60 minutes data fusion intervals

Figure 10 illustrates the real field energy forecasting results for 30 and 60 minutes time step cases. Figure 10a shows the forecasting results with 30 minutes interval, and Figure 10b is for the models with 60 minutes interval. Even though the Kalman filter is able to improve the accuracy to some extent, the final results are still not as good as the results from 15 minutes forecasting interval. As a result, the data fusion interval plays a critical role in the final results. The more frequent the model is updated, the higher the accuracy the model can achieve. However, the model updating needs data collecting, storage, and computing effort. So a balance between accuracy and updating frequency should be determined based on the desired performance and affordable efforts. 

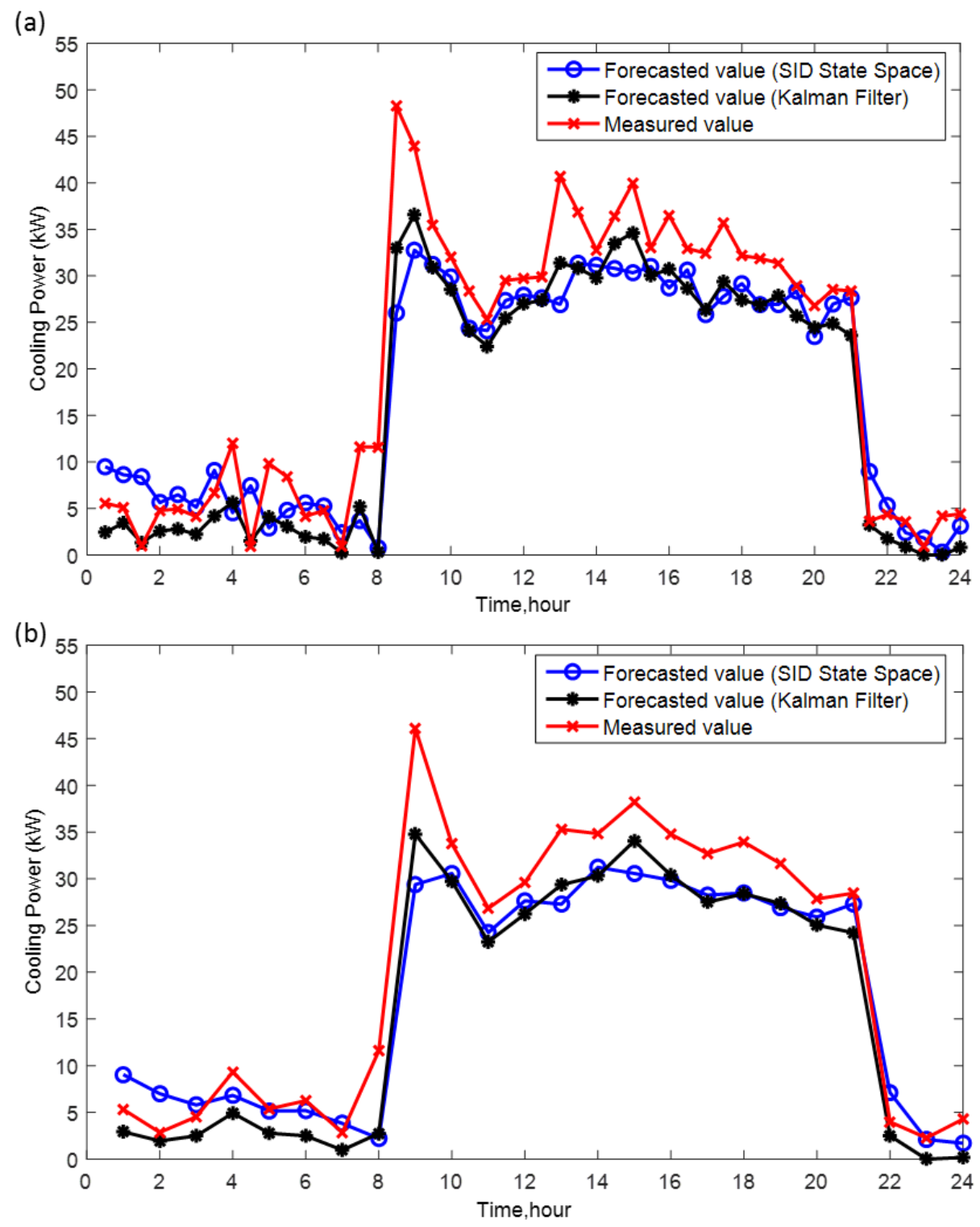

Figure 10. Real building energy forecasting model calibration results

In order to show the effectiveness of the real field model on-line adaptation, Table 5 compares the real filed energy forecasting model performance from all these three models with different time steps. Similar to the conclusion in the virtual building study, the state space models loss certain accuracy when comparing with the original SID models, and using the Kalman filter technique with the state space model improves the model performance. The level of improvement is affected by the measurement accuracy and the data fusion intervals. Even in the worst case with 60-minute updating intervals, the Kalman filter model is still able to achieve $87 \%$ accuracy $\left(\mathrm{R}^{2}\right)$, which is still acceptable for building control and 
operation. Similar to the virtual building cases, the fractional bias indexes are also evaluated in the real building cases. The overall findings are slightly different to those in the virtual building cases. FB indexes are below zero in all the cases, and that from Kalman filter models are closer to zero comparing with the SID and state space models.

Table 5. Real building energy forecasting accuracy comparison

\begin{tabular}{cccccc}
\hline Interval, min & Model & $\mathbf{R}^{\mathbf{2}}$ & RMSE, $\mathbf{k W}$ & NRMSE & FB \\
\hline \multirow{2}{*}{15} & SID & 0.85 & 4.8 & $10.2 \%$ & -0.17 \\
& State Space & 0.79 & 6.1 & $14.0 \%$ & -0.23 \\
& Kalman Filter & $\mathbf{0 . 9 2 *}$ & $\mathbf{3 . 9}$ & $\mathbf{7 . 0 \%}$ & $\mathbf{- 0 . 1 5}$ \\
\hline \multirow{2}{*}{30} & SID & 0.82 & 10.9 & $14.0 \%$ & -0.24 \\
& State Space & 0.72 & 12.5 & $15.3 \%$ & -0.28 \\
& Kalman Filter & $\mathbf{0 . 8 6}$ & $\mathbf{4 . 9}$ & $\mathbf{1 0 . 0 \%}$ & $\mathbf{- 0 . 2 2}$ \\
\hline \multirow{2}{*}{60} & SID & 0.76 & 12.9 & $15.0 \%$ & -0.25 \\
& State Space & 0.70 & 13.4 & $16.0 \%$ & -0.29 \\
& Kalman Filter & $\mathbf{0 . 8 7}$ & $\mathbf{4 . 6}$ & $\mathbf{1 1 . 0 \%}$ & $\mathbf{- 0 . 1 9}$ \\
\hline
\end{tabular}

*Highest accuracy $\left(\mathrm{R}^{2}\right)$, lowest error (RMSE and NRMSE), and FB indexes closest to zero are bold

\section{Conclusion}

A new systematic methodology, which is based on frequency response system identification and data fusion technique, is introduced in this study for on-line building energy estimation model development. Frequency response function model realized by power spectral density model is firstly used to develop a SID model to forecast building cooling energy consumption. ERA is then developed and implemented to convert the energy forecasting models to be state space models. Finally, Kalman filter technique is applied on these state space models to improve the forecasting accuracy and robustness. Three intervals, i.e., 15, 30 and 60 minutes, are tested in this study. The proposed on-line energy forecasting methodology is validated in a virtual building and a real commercial building. In the real field implementation, the Kalman filters update and adapt the energy forecasting models based on the measurements at each time step. The overall building energy estimation accuracy from this proposed method can reach to above $95 \%$ in the virtual building case and around $90 \%$ in the real building case within 2 minutes calculation time. Future efforts are needed to examine the developed methodologies in different types of buildings and 
HVAC systems as well as to include energy consumption from heating, lighting, and electrical appliance, etc, into this forecasting model.

\section{Acknowledgments}

Financial support provided by the U.S. National Science Foundation Award 1239247 is greatly appreciated. The authors also would like to acknowledge Dr. Ran Liu and Dr. Xiaohui Zhou from Iowa Energy Center for their help in the real field study. X. Li is grateful the Postdoctoral Fellowship from The Center for Green Buildings and Cities (CGBC) at Harvard University.

\section{Reference}

1. DOE, U.S. Buildings Energy Data Book, http://buildingsdatabook.eren.doe.gov/. 2013 [cited 2015 01.10].

2. Samuelson, H., et al., Parametric energy simulation in early design: High-rise residential buildings in urban contexts. Building and Environment, 2016. 101: p. 19-31.

3. Ascione, F., et al., Multi-stage and multi-objective optimization for energy retrofitting a developed hospital reference building: A new approach to assess cost-optimality. Applied Energy, 2016. 174: p. 37-68.

4. Malkawi, A., et al., Predicting thermal and energy performance of mixed-mode ventilation using an integrated simulation approach. Building Simulation, 2016: p. 1-12.

5. Tong, Z., et al., Quantifying the impact of traffic-related air pollution on the indoor air quality of a naturally ventilated building. Environment International, 2016. 89-90: p. 138-146.

6. $\quad \mathrm{Hu}, \mathrm{M} ., \mathrm{J} . \mathrm{D}$. Weir, and T. Wu, Decentralized operation strategies for an integrated building energy system using a memetic algorithm. European Journal of Operational Research, 2012. 217(1): p. 185-197.

7. Li, X., J. Wen, and A. Malkawi, An operation optimization and decision framework for a building cluster with distributed energy systems. Applied Energy, 2016. 178: p. 98-109.

8. Privara, S., et al., Building modeling as a crucial part for building predictive control. Energy and Buildings, 2013. 56: p. 8-22.

9. Li, X. and J. Wen, Review of building energy modeling for control and operation. Renewable and Sustainable Energy Reviews, 2014. 37: p. 517-537.

10. Office of Energy Efficiency and Renewable Energy, U.S.D.o.E. EnergyPlus Energy Simulation Software: About EnergyPlus. [cited 2014 March 03]; Available from: http://www.energyplus.gov.

11. May-Ostendorp, P., et al., Model-predictive control of mixed-mode buildings with rule extraction. Building and Environment, 2011. 46(2): p. 428-437.

12. Coffey, B., et al., A software framework for model predictive control with GenOpt. Energy and Buildings, 2010. 42(7): p. 1084-1092.

13. Seo, D., P. Ihm, and M. Krarti, Development of an optimal daylighting controller. Building and Environment, 2011. 46(5): p. 1011-1022.

14. Comodi, G., et al., Multi-apartment residential microgrid with electrical and thermal storage devices: experimental analysis and simulation of energy management strategies. Applied Energy, 2015. 137: p. 854-866.

15. Feng, J.D., et al., Model predictive control of radiant slab systems with evaporative cooling sources. Energy and Buildings, 2015. 87: p. 199-210. 
16. Avci, M., et al., Model predictive HVAC load control in buildings using real-time electricity pricing. Energy and Buildings, 2013.

17. Yan, B. and A.M. Malkawi. A Bayesian approach for predicting building cooling and heating consumption. in Proceedings of 13th International Building Performance Simulation Association Conference. 2013.

18. Moon, J.W., Performance of ANN-based predictive and adaptive thermal-control methods for disturbances in and around residential buildings. Building and Environment, 2012. 48: p. 15-26.

19. Xi, X.-C., A.-N. Poo, and S.-K. Chou, Support vector regression model predictive control on a HVAC plant. Control Engineering Practice, 2007. 15(8): p. 897-908.

20. Cui, C., et al., Short-term building energy model recommendation system: A meta-learning approach. Applied Energy, 2016. 172: p. 251-263.

21. Eisenhower, B., et al., A methodology for meta-model based optimization in building energy models. Energy and Buildings, 2012. 47: p. 292-301.

22. Braun, J.E. and N. Chaturvedi, An inverse gray-box model for transient building load prediction. HVAC\&R Research, 2002. 8(1): p. 73-99.

23. Ji, Y., et al., Estimating hourly cooling load in commercial buildings using a thermal network model and electricity submetering data. Applied Energy, 2016. 169: p. 309-323.

24. Oldewurtel, F., et al., Use of model predictive control and weather forecasts for energy efficient building climate control. Energy and Buildings, 2012. 45: p. 15-27.

25. Li, X. and J. Wen, Building energy consumption on-line forecasting using physics based system identification. Energy and Buildings, 2014. 82: p. 1-12.

26. Li, X., J. Wen, and E.-W. Bai, Developing a whole building cooling energy forecasting model for on-line operation optimization using proactive system identification. Applied Energy, 2016. 164: p. 69-88.

27. $\mathrm{Li}, \mathrm{X}$., et al., Commercial building cooling energy forecasting using proactive system identification: A whole building experiment study. Science and Technology for the Built Environment, 2016.

28. Maasoumy, M., et al., Handling model uncertainty in model predictive control for energy efficient buildings. Energy and Buildings, 2014. 77: p. 377-392.

29. $\mathrm{Hu}, \mathrm{M}$., A data-driven feed-forward decision framework for building clusters operation under uncertainty. Applied Energy, 2015. 141: p. 229-237.

30. Juang, J.-N. and R.S. Pappa, An eigensystem realization algorithm for modal parameter identification and model reduction. Journal of guidance, control, and dynamics, 1985. 8(5): p. 620-627.

31. Deru, M., et al., US Department of Energy commercial reference building models of the national building stock. 2011.

32. Domínguez-Muñoz, F., J.M. Cejudo-Lopez, and A. Carrillo-Andrés, Uncertainty in peak cooling load calculations. Energy and Buildings, 2010. 42(7): p. 1010-1018.

33. Li, X., J. Wen, and T. Wu, Net-zero Energy Impact Building Clusters Emulator for Operation Strategy Development, in 2014 ASHRAE Annual Conference 2014: Seattle, WA. 